\title{
Self-Reported Cognitive Decline Based on the Ascertain Dementia 8 Questionnaire May Be Effective for Detecting Mild Cognitive Impairment and Dementia in the Community: The Wakuya Project
}

\author{
Mari Kasai ${ }^{\mathrm{a}}$ Tomohiro Sugawara $^{\mathrm{a}}$ Junko Takada ${ }^{\mathrm{a}}$ Keiichi Kumai ${ }^{\mathrm{a}}$ Kei Nakamura ${ }^{\mathrm{a}}$ \\ Kenichi Meguro ${ }^{a, b}, c$ \\ aGeriatric Behavioral Neurology Project, New Industry Creation Hatchery Center, Tohoku University, Sendai, Japan; \\ ${ }^{b}$ Cyclotron Radioisotope Center, Tohoku University, Sendai, Japan; ' Tohoku University Graduate School of Medicine, Sendai, \\ Japan
}

\section{Keywords}

AD8 · Dementia $\cdot$ Mild cognitive impairment · Early detection

\begin{abstract}
Introduction: To assess cognitive impairment, self-awareness is an important issue. The Ascertain Dementia 8 questionnaire (AD8) is a brief observation checklist for detecting mild cognitive impairment ( $\mathrm{MCl}$ ) and dementia. After analyzing the reliability and validity of a self-reported Japanese version of the AD8 (AD8-J), we compared self- and informantreported versions of the AD8-J. Methods: A total of 93 community residents aged 75 years or older living in Wakuya, Northern Japan, agreed to participate in this study; 35 were rated as Clinical Dementia Rating (CDR) 0 (healthy), 46 as CDR 0.5 (defined herein as $\mathrm{MCI}$ ), and 12 as CDR 1 or above (dementia, confirmed by the DSM-IV). We examined the reliability and validity using a receiver operating characteristic (ROC) curve. We analyzed the differences between self-reported and informant-reported AD8-J using a repeated measures ANOVA. Results: The self-reported AD8-J showed a satisfactory reliability (i.e., Cronbach coefficient, $a=0.71$; Guttman split half method coefficient $=0.60$ ). For CDR 0 vs. CDR
\end{abstract}

0.5 or above, the area under the ROC curve was 0.74 and the cutoff score was $1 / 2$, with a sensitivity of $70.7 \%$ and a specificity of $65.7 \%$. Analysis of the subscores of AD8 suggested that, from the early stage of dementia, the subjects showed a subjective decline in memory and interest in hobbies/activities, as well as problems with judgment. Conclusion: It is suggested that the self-reported AD8-J was effective in detecting $\mathrm{MCl}$ and dementia. We could use it for detecting $\mathrm{MCl}$ and dementia, including in those living alone, in the primary health checkup.

(c) 2021 The Author(s)

Published by S. Karger AG, Basel

\section{Introduction}

The stage of Clinical Dementia Rating (CDR) 0.5 is the borderline condition between health and dementia [1]. Previously, we reported that the prevalence of amnestic mild cognitive impairment (MCI) was $4.9 \%$, whereas that of CDR 0.5 was $30.2 \%$ [2]; thus we considered that the concept of CDR 0.5 was better as a screen for participants with prodromal dementia than amnestic MCI.
(C) 2021 The Author(s)

Published by S. Karger AG, Basel

This is an Open Access article licensed under the Creative Commons Attribution-NonCommercial-4.0 International License (CC BY-NC) (http://www.karger.com/Services/OpenAccessLicense), applicable to the online version of the article only. Usage and distribution for commercial purposes requires written permission.
Kenichi Meguro

Geriatric Behavioral Neurology Project

New Industry Creation Hatchery Center, Tohoku University

IDAC, 4-1, Seiryo-machi, Aoba-ku, Sendai 980-8575 (Japan)

kenichi.meguro.e6@ tohoku.ac.jp 
Table 1. Demographics of the participants

\begin{tabular}{lllrrr}
\hline & $\begin{array}{l}\text { CDR 0 } \\
\text { (healthy elderly) }\end{array}$ & $\begin{array}{l}\text { CDR 0.5 } \\
\text { (MCI) }\end{array}$ & $\begin{array}{l}\text { CDR 1+ } \\
\text { (dementia) }\end{array}$ & $F / \chi^{2}$ value & $p$ value \\
\hline Patients (male/female ratio), $n$ & $35(11 / 24)$ & $46(19 / 27)$ & $12(5 / 7)$ & 0.9 & 0.631 \\
Age, years & $80.0(4.1)$ & $80.2(4.0)$ & $83.8(3.4)^{\mathrm{ab}}$ & 4.4 & 0.015 \\
Education, years & $11.0(2.0)$ & $10.6(2.5)$ & $9.1(2.6)$ & 3.0 & 0.055 \\
MMSE score & $25.9(2.6)$ & $23.7(3.0)^{\mathrm{a}}$ & $20.2(3.5)^{\mathrm{ab}}$ & 18.1 & $<0.001$ \\
\hline
\end{tabular}

Values are presented as means (SD) unless otherwise stated. Statistical analyses: sex, $\chi^{2}$ test; other variables,

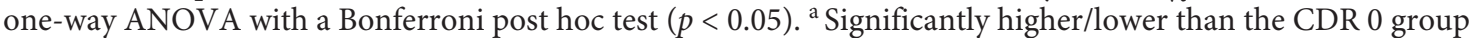
$(p<0.05){ }^{\mathrm{b}}$ Significantly higher/lower than the CDR 0.5 group $(p<0.05)$.

However, the scoring of informant-based instruments such as the CDR is dependent on the reliability of the family or caregiver [3]. According to information from the government, in 2016 the number of 1-person households with persons aged 65 or older was $27 \%$ [4]. For elderly people who live alone, we could not use an informant rating assessment.

Alternative to an informant-based approach, there are performance-based tests and self-reported tests. The former include neuropsychological tests such as the MiniMental State Examination (MMSE) [5]; however, these tests may be difficult to use to evaluate recent changes [3].

Self-rating scales were not so frequently used, since many dementia patients have a lack of awareness or denial of cognitive deficits (cognitive anosognosia). Awareness of cognitive impairments varies between individuals, with some patients offering reliable accounts of cognitive change and others failing to estimate symptoms $[3,6]$. Meanwhile, some patients with dementia have been reported to be able to self-rate the physical and psychological symptoms such as depression [7].

There are several self-rating tests for detecting MCI and dementia, such as the Ascertain Dementia 8 questionnaire (AD8) [8], the Self-Administered Gerocognitive Examination (SAGE) [9], the 64-item Memory Functioning Questionnaire [10,11], and the Self-Administered Dementia Checklist [12].

The AD8 consists of 8 short questions about memory complaints. Galvin et al. [8] reported the use of the selfreported AD8 as an alternative scale of informant-reported AD8. The SAGE was developed as a 10- to 15-min selfadministered cognitive assessment tool, but it was affected by the individual's low educational attainment and visual impairment $[3,9]$. The 64-item Memory Functioning Questionnaire consisted of 64 items of everyday memory functioning $[10,11]$, but it was too long for general use.

The self-reported AD8 was reported to have good sensitivity and specificity for distinguishing MCI and dementia patients from healthy populations [8]. Since the self-reported AD8 includes objective questions about everyday functional activities in a nonthreatening fashion [8], it might be suitable for assessing MCI and dementia.

Since there were no previous studies of a Japanese version of the self-reported AD8 (Self-AD8-J), the first aim of this study was to establish its reliability and validity. The second aim was to compare self- and informant-reported versions to discuss self-awareness of cognitive impairment.

\section{Methods}

\section{Participants}

From December 2017 to March 2018, one hundred residents ( 35 men and 58 women) aged 75 years or older living in Wakuya, Northern Japan, agreed to participate in this study. Ninety-three participants completed the Self-AD8-J and the Japanese version of the informant-reported AD8 (Info-AD8-J); 35 (11 men and 24 women) were rated as CDR 0 (healthy), 46 (19 men and 27 women) as CDR 0.5 (questionable dementia or very mild dementia), and 12 (5 men and 7 women) as CDR 1 or higher (dementia; Table 1).

We excluded 7 participants due to incomplete data sets. All subject with CDR 1 or higher met the criteria for a dementia diagnosis with the DSM-IV [13]. We also conducted clinical examinations and neuropsychological tests. With regard to demographic data, significant differences were found among the 3 CDR groups in terms of age, educational level, and MMSE scores using a oneway ANOVA and Bonferroni post hoc group comparisons; $p<$ 0.05 was considered statistically significant.

\section{Assessments}

Ascertain Dementia 8 Questionnaire

The informant-reported $\operatorname{AD} 8[8,14,15]$ is a brief and sensitive informant-based assessment for detection of participants with 
MCI and dementia in a community. The informant-reported AD8 consists of 8 short questions about judgement and memory complaints, with 3 answer options each, and the estimated time is $2-3$ min. The 3 answer options of the AD8 include: (1) yes (change), (2) no (no change), and (3) N/A (do not know).

In the informant-reported AD8, the cutoff score of the original version for screening for MCI and dementia was $1 / 2$, with a sensitivity of $84 \%$ and a specificity of $93 \%$ [8]. Meanwhile, the cutoff score of the Info-AD8-J for screening for dementia was $1 / 2$, with a sensitivity of $88 \%$ and a specificity of $68 \%$ in a community-based study [16].

In the self-reported $\mathrm{AD} 8$, the cutoff score of the original version for screening for MCI and dementia was $0 / 1$, with a sensitivity of $80 \%$ and a specificity of $59 \%$ [8].

We used the Self-AD8-J and the Info-AD8-J [16].

\section{Clinical Dementia Rating}

A clinical team comprised of skilled physicians (2 neurologists and 2 psychiatrists) and public health nurses determined the CDR for each participant $[1,17]$ and were blinded to the cognitive test results. They used the Japanese version of the questionnaire of the CDR work sheet [18]. The CDR contains the following 6 domains: memory, orientation, judgment and problem solving, community affairs, home and hobbies, and personal care. Before the interviews with the physicians, the public health nurses visited the participants' homes to evaluate their daily activities. Finally, with reference to the information provided by the family members, the CDR for each of the participants was determined at a joint meeting of the physicians and public health nurses. One author (K.M.) was certified as a CDR rater by the Knight Alzheimer's Disease Research Center's Memory and Aging Project at the Washington University School of Medicine.

\section{Mini-Mental State Examination}

We used the MMSE [5] to assess general cognitive functions. The MMSE consists of orientation, registration, serial 7s, recall, naming, repetition, comprehension, reading, writing, and drawing. Scores on the MMSE range from 0 (poor) to 30 (good).

Analyses

Reliability of the Self-AD8-J

We used the Cronbach coefficient $\alpha$ and the Guttman split half method coefficient for reliability analysis of the Self-AD8-J.

Validity of Self-AD8-J and the Info-AD8-J

We calculated the area under the curve (AUC) of the receiver operating characteristic (ROC) curve and its 95\% CI for the SelfAD8-J score, the Info-AD8-J, and the MMSE (CDR 0 vs. CDR 0.5 or higher [CDR 0.5+]). Participants with a CDR of $0.5+$ included those with a CDR of $0.5(n=46)$ and those with a CDR of $1+(n=$ 12). Sensitivity, specificity, positive predictive values and negative predictive values, as well as likelihood ratios, were calculated with standard formulas. We compared the AUC of Self-AD8-J and Info-AD8-J scores with that of the MMSE using paired Z-score analysis [19].

Differences between the Self-AD8-J and the Info-AD8-J

We analyzed the differences between the Self-AD8-J and the Info-AD8-J among the $3 \mathrm{CDR}$ groups using a repeated measures ANOVA (2 rater effects [Self-AD8-J vs. Info-AD8-J] × 3 CDR ef-

Self-Reported AD8 in Japan

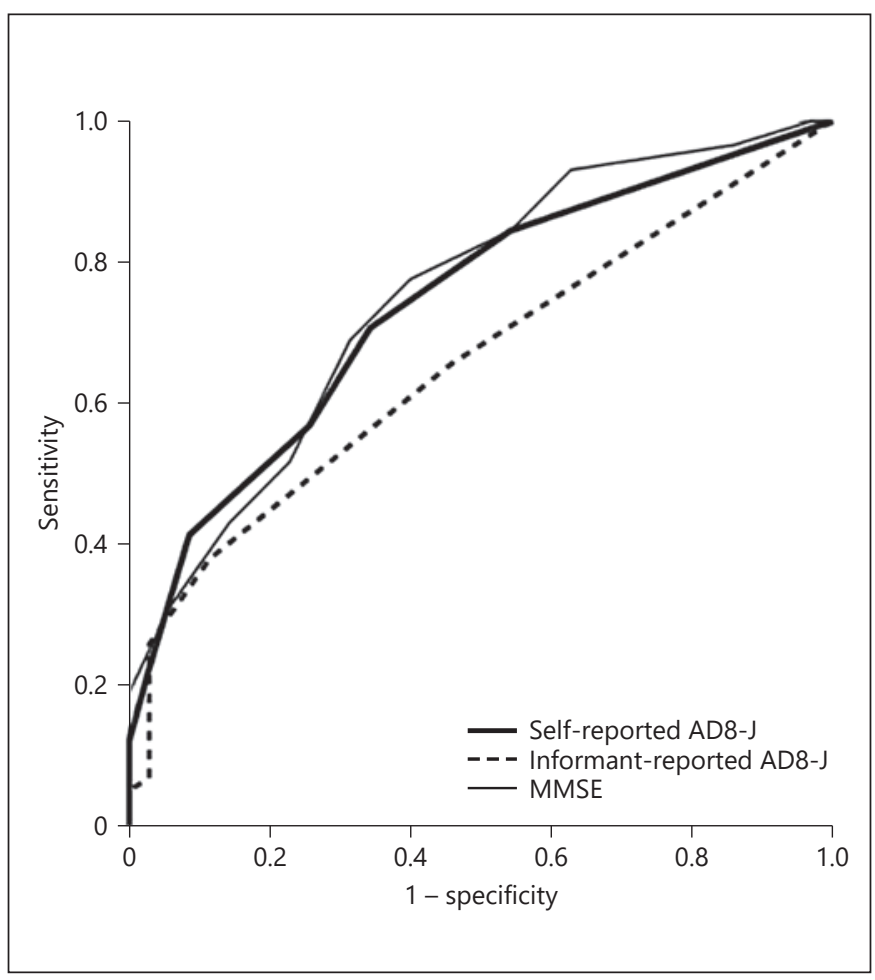

Fig. 1. ROC of Self-AD8-J and the Info-AD8-J and the MMSE: CDR 0 vs. CDR 0.5 or higher. Thick line: ROC of the Self-AD8-J. Dashed line: ROC of the Info-AD8-J. Thin line: ROC of the MMSE.

fects [CDR 0 vs. CDR 0.5 vs. CDR $1+$ ]) with a Bonferroni post hoc test (significance level: $p<0.05$ ). When there was a significant interaction between these effects, we used the $t$ test for rater effect and a one-way ANOVA with a Bonferroni post hoc test for CDR effect (significance level: $p<0.05$ ).

We used IBM SPSS statistics version 22 software (IBM, Corp., Armonk, NY, USA) for all statistical analyses in this study.

\section{Results}

\section{Reliability of the Self-AD8-J}

The Cronbach a coefficient was 0.71 (range 0.65-0.72), and the Guttman split half method coefficient was 0.60 (group A: items 1, 2, 3, and 4; group B: items 5, 6, 7, and 8).

\section{Validity of the Self-AD8-J and the Info-AD8-J}

Figure 1 shows the ROC curves of the Self-AD8-J and the Info-AD8-J and the MMSE.

Table 2 shows the ROC curve and cutoff scores and diagnostic accuracy, sensitivity, and specificity of the SelfAD8-J and the Info-AD8-J. 
Table 2. ROC curve and diagnostic accuracy of the Self-AD8-J and the Info-AD8-J

\begin{tabular}{|c|c|c|}
\hline & \multicolumn{2}{|c|}{$\begin{array}{l}\text { CDR } 0 \text { vs. CDR } \\
0.5 \text { or higher }\end{array}$} \\
\hline Patients, $n$ & \multicolumn{2}{|c|}{35 vs. 58} \\
\hline \multicolumn{3}{|l|}{$\operatorname{AUC}(95 \% \mathrm{CI})$} \\
\hline Self-AD8-J & \multicolumn{2}{|c|}{$0.74(0.64-0.84), p<0.001$} \\
\hline Info-AD8-J & \multicolumn{2}{|c|}{$0.66(0.55-0.77), p=0.012$} \\
\hline MMSE & \multicolumn{2}{|c|}{$0.75(0.65-0.85), p<0.001$} \\
\hline \multicolumn{3}{|l|}{ Z-score } \\
\hline Self-AD8-J vs. MMSE & \multicolumn{2}{|c|}{$Z=-0.15, p=0.444$} \\
\hline Info-AD8-J vs. MMSE & \multicolumn{2}{|c|}{$\mathrm{Z}=-1.08, p=0.140$} \\
\hline Self-AD8-J vs. Info-AD8-J & \multicolumn{2}{|c|}{$Z=0.98, p=0.164$} \\
\hline \multicolumn{3}{|c|}{ Diagnostic accuracy of the Self-AD8-J } \\
\hline Cutoff score & $0 / 1$ & $1 / 2$ \\
\hline Sensitivity, \% & 85.5 & 70.7 \\
\hline Specificity, \% & 45.7 & 65.7 \\
\hline Positive predictive value, $\%$ & 72.1 & 77.4 \\
\hline Negative predictive value, $\%$ & 64.0 & 57.5 \\
\hline Positive likelihood ratio & 1.56 & 2.06 \\
\hline Negative likelihood ratio & 0.34 & 0.45 \\
\hline \multicolumn{3}{|c|}{ Diagnostic accuracy of the Info-AD8-J } \\
\hline Cutoff score & $0 / 1$ & $1 / 2$ \\
\hline Sensitivity, \% & 65.5 & 37.9 \\
\hline Specificity, \% & 54.3 & 88.6 \\
\hline Positive predictive value, $\%$ & 70.4 & 84.6 \\
\hline Negative predictive value, $\%$ & 48.7 & 46.3 \\
\hline Positive likelihood ratio & 1.43 & 3.32 \\
\hline Negative likelihood ratio & 0.64 & 0.70 \\
\hline
\end{tabular}

\section{Differences between the Self-AD8-J and the Info-AD8-J}

Table 3 shows the results of the mean total and subscores of the Self-AD8-J and the Info-AD8-J among the CDR groups. The total score and those of items 2, 3, 7, and 8 showed significant interactions between rater and CDR effects.

Total score: rater and CDR effects with an interaction.

- Rater effect: the Self-AD8-J score was severer than the Info-AD8-J score in the CDR 0.5 group.

- CDR effect: the Self-AD8-J scores of the CDR 0.5 and $1+$ groups were severer than that of the CDR 0 group. The Info-AD8-J score of the CDR 1+ group was severer than those of the CDR 0 and 0.5 groups.

Item 1 (problems with judgment): rater and CDR effects, but no interaction.

- Rater effect: the Self-AD8-J score was severer than the Info-AD8-J score.

- CDR effect: the score of the CDR 1+ group was severer than that of the CDR 0 group.
Item 2 (reduced interest in hobbies/activities): rater and CDR effects with an interaction.

- Rater effect: the Self-AD8-J score was severer than the Info-AD8-J score in the CDR 0.5 group.

- CDR effect: the Self-AD8-J scores of the CDR 0.5 and $1+$ groups were severer than that of the CDR 0 group. The Info-AD8-J score of the CDR 1+ group was severer than those of the CDR 0 and 0.5 groups.

Item 3 (repeats questions, stories, or statements): CDR effect with an interaction, but no rater effect.

- Rater effect: the Self-AD8-J score was severer than the Info-AD8-J score in the CDR 0.5 group.

- CDR effect: there was no difference among the 3 groups in terms of the Self-AD8-J score. The InfoAD8-J score of the CDR 1+ group was severer than those of the CDR 0 and 0.5 groups.

Item 4 (trouble learning how to use tools, appliances, or gadgets): no rater or CDR effects with no interaction. Item 5 (forgets the correct month or year): CDR effect, but no rater effect with no interaction.

- CDR effect: the score of the CDR 1+ group was severer than those of the CDR 0 and 0.5 groups.

Item 6 (difficulty handling complicated financial affairs): CDR effect, but no rater effect with no interaction.

- CDR effect: the score of CDR 1+ group was severer than those of the CDR 0 and 0.5 groups.

Item 7 (difficulty remembering appointments): rater and CDR effects with an interaction.

- Rater effect: the Info-AD8-J score was severer than the Self-AD8-J score in the CDR 1+ group.

- CDR effect: there was no difference among the 3 groups in terms of the Self-AD8-J score. The InfoAD8-J score of the CDR $1+$ group was severer than those of the CDR 0 and 0.5 groups.

Item 8 (consistent problems with thinking and/or memory): rater and CDR effects with an interaction.

- Rater effect: the Self-AD8-J score was severer than the Info-AD8-J score in the CDR 0.5 group.

- CDR effect: the Self-AD8-J score of the CDR 0.5 group was severer than that of the CDR 0 group. The InfoAD8-J score of the CDR 1+ group was severer than those of the CDR 0 and 0.5 groups.

\section{Discussion}

\section{Reliability of the Self-AD8-J}

Since the previous study reported that Cronbach $a$ values of $0.7-0.8$ are regarded as satisfactory [20], we considered that the Self-AD8-J had enough reliability for analysis. 
Table 3. Self-AD8-J and Info-AD8-J scores

\begin{tabular}{|c|c|c|c|c|c|c|c|c|c|}
\hline & \multicolumn{2}{|c|}{ CDR 0 (healthy elderly) } & \multicolumn{2}{|c|}{ CDR 0.5 (MCI) } & \multicolumn{2}{|c|}{ CDR 1+ (dementia) } & \multirow{2}{*}{$\begin{array}{l}\text { Rater effect } \\
F \text { value }\end{array}$} & \multirow{2}{*}{$\begin{array}{l}\text { CDR effect } \\
F \text { value }\end{array}$} & \multirow{2}{*}{$\begin{array}{l}\text { Interaction } \\
F \text { value }\end{array}$} \\
\hline & Self-AD8-J & Info-AD8-J & Self-AD8-J & Info-AD8-J & Self-AD8-J & Info-AD8-J & & & \\
\hline Total score & $1.3(1.5)$ & $0.7(1.1)$ & $2.8(1.9)^{\mathrm{ac}}$ & $1.1(1.3)$ & $3.4(2.3)^{b}$ & $4.2(2.6)^{\mathrm{ab}}$ & 4.7 & 20.4 & 8.8 \\
\hline \multicolumn{10}{|l|}{ Subscore } \\
\hline Item 1 & $0.3(0.4)^{\mathrm{c}}$ & $0.0(0.2)$ & $0.4(0.5)^{\mathrm{c}}$ & $0.1(0.3)$ & $0.6(0.5)^{\mathrm{ac}}$ & $0.3(0.5)^{\mathrm{a}}$ & 17.9 & 4.5 & 0.1 \\
\hline Item 4 & $0.3(0.5)$ & $0.3(0.5)$ & $0.5(0.5)$ & $0.3(0.5)$ & $0.3(0.5)$ & $0.5(0.5)$ & 0.01 & 0.8 & 1.6 \\
\hline Item 5 & $0.1(0.3)$ & $0.1(0.2)$ & $0.3(0.5)^{\mathrm{a}}$ & $0.1(0.2)^{\mathrm{a}}$ & $0.6(0.5)^{\mathrm{a}}$ & $0.7(0.5)^{\mathrm{a}}$ & 0.8 & 28.8 & 2.3 \\
\hline Item 6 & $0.0(0.0)$ & $0.0(0.0)$ & $0.1(0.3)^{\mathrm{a}}$ & $0.0(0.1)^{\mathrm{a}}$ & $0.2(0.4)^{\mathrm{a}}$ & $0.3(0.5)^{\mathrm{a}}$ & 0.2 & 8.2 & 1.1 \\
\hline Item 7 & $0.0(0.2)$ & $0.0(0.0)$ & $0.1(0.3)$ & $0.0(0.2)$ & $0.1(0.3)^{\mathrm{d}}$ & $0.5(0.5)^{\mathrm{ab}}$ & 6.4 & 8.5 & 11.6 \\
\hline Item 8 & $0.2(0.4)$ & $0.1(0.3)$ & $0.5(0.5)^{\mathrm{ac}}$ & $0.1(0.3)$ & $0.6(0.5)$ & $0.6(0.5)^{\mathrm{ab}}$ & 6.0 & 9.4 & 3.4 \\
\hline
\end{tabular}

Values are presented as means (SD) unless otherwise stated. Statistical analysis: repeated measures ANOVA (2 rater effects [SelfAD8-J vs. Info-AD8-J] $\times 3$ CDR effects [CDR 0 vs. CDR 0.5 vs. CDR 1 or higher]; significance level: $p<0.05)^{\text {a }}$ Significantly higher/ lower than the CDR 0 group $(\mathrm{p}<0.05)$. ${ }^{\mathrm{b}}$ Significantly higher/lower than the CDR 0.5 group $(p<0.05)$. ${ }^{\mathrm{c}}$ Significantly higher than the Info-AD8-J group $(p<0.05)$. ${ }^{\mathrm{d}}$ Significantly lower than the Info-AD8-J group $(p<0.05)$.

Validity of the Self-AD8-J and the Info-AD8-J

If the cutoff scores of the Self-AD8-J were 0/1, then the sensitivity would be good but the specificity would be poor for detecting MCI and dementia. In contrast, if the cutoff level of the Self-AD8-J were 1/2, then the sensitivity would be lower and the specificity higher than the cutoff level of $0 / 1$ in the study of Galvin et al. [20]. Our results regarding the Self-AD8-J suggest that the "subjective" memory complaints of MCI participants may be a common feature across cultures (e.g., St. Louis, MO, USA, and Wakuya, Japan).

If the cutoff scores of the Info-AD8-J were $0 / 1$, then the sensitivity and the specificity would be poor for detecting MCI and dementia. In contrast, if the cutoff were $1 / 2$, then the specificity would be higher. The town of Wakuya promotes the longevity of elderly people, and we have organized the Strategy Meeting on Dementia Prevention with town officials. We thought that our education effort should be gradually widespread so as to increase the ability to detect early dementia in the community through observation.

\section{Differences between the Self-AD8-J and the \\ Info-AD8-J}

There was an interaction in the total and items $2,3,7$, and 8. In item 2, CDR 0.5 participants had a subjective decline in "interest in hobbies/activities." In a previous study, elderly people subjectively felt a reducing of QOL when they actually had objective home and hobby impairment [21]. The subjective decline in hobbies and ac- tivities may be an assessment point for early detection of MCI and dementia.

In items 3 and 8, i.e., "unneeded repetition of questions" and "consistent problems with thinking and/or memory," some CDR 0.5 participants had subjective memory complaints; however, their family members might not have recognize mild cognitive changes.

In item 7, clinically we know that "forgetting of an appointment" for a hospital visit is an objective symptom of early-stage dementia. Dementia patients may have cognitive anosognosia with regard to their severe cognitive impairments $[3,21]$, and their caregivers may overestimate their symptoms.

In item 1, i.e., "problems with judgment," the score on the Self-AD8-J was severer than those on the Info-AD8-J. The score of the CDR $1+$ group was severer than those of the CDR 0 and 0.5 groups for item 5 , i.e., "forgets the correct month or year," and item 6, i.e., "difficulty handling complicated financial affairs."

There were no significant rater or CDR effects on item 4, i.e., "trouble learning how to use tools, appliances, or gadgets" in this study. About $30 \%$ of the healthy elderly had difficulty learning how to use these appliances since newer-model home electrical appliances tend to have a high degree of performance and complexity.

\section{Limitations}

This study has several limitations. The first limitation is that our sample size of patients with dementia was 
small. We could not conduct a test-retest reliability analysis since this study had a community-based design. We used only the MMSE as a neuropsychological test for validity analysis. It may be difficult to detect MCI in healthy elderly populations with a high educational attainment, as in this study.

\section{Conclusions}

This study indicated that the Self-AD8-J had a good sensitivity for detecting MCI and dementia. It is suggested that the Self-AD8-J is superior in detecting MCI and dementia. We could use the Self-AD8-J for detecting MCI and dementia, including in those living alone, in the primary health checkup.

\section{Acknowledgment}

We are grateful to all of the staff in Wakuya, especially the public health nurses, and the staff at the Geriatric Behavioral Neurology Project, Tohoku University New Industry Creation Hatchery Center (NICHe). In particular, we thank Ms. Keiko Chida, Yuriko Kato, and Megumi Kano.

\section{Statement of Ethics}

Written informed consent was obtained from each of the participants and their families. This study was approved by the Ethical Committees of the Wakuya government and the Tohoku University Graduate School of Medicine (2014-1-565).

\section{Conflict of Interest Statement}

The authors have no conflict of interests to declare.

\section{Funding Sources}

This work was supported by the Commissioned Research Fund of the City of Wakuya.

\section{Author Contributions}

M.K.: data analysis and writing of this paper. K.M.: design, direction, and writing of this paper. T.S., K.K., J.T., and K.N.: data collection.

\section{References}

1 Morris JC. The Clinical Dementia Rating (CDR): current version and scoring rules. Neurology. 1993 Nov;43(11):2412-4.

2 Meguro K, Ishii H, Yamaguchi S, Ishizaki J, Sato M, Hashimoto R, et al. Prevalence and cognitive performances of clinical dementia rating 0.5 and mild cognitive impairment in Japan. The Tajiri project. Alzheimer Dis Assoc Disord. 2004 Jan-Mar;18(1):3-10.

3 Galvin JE. Using Informant and Performance Screening Methods to Detect Mild Cognitive Impairment and Dementia. Curr Geriatr Rep. 2018 Mar;7(1):19-25.

4 Cabinet Office Japan. The Ageing Society: Current Situation and Implementation Measures FY 2017 [Internet]. Available from: https://www8.cao.go.jp/kourei/english/annualreport/2018/pdf/c1-1.pdf.

5 Folstein MF, Folstein SE, McHugh PR. "Minimental state". A practical method for grading the cognitive state of patients for the clinician. J Psychiatr Res. 1975 Nov;12(3):189-98.

6 Kelleher M, Tolea MI, Galvin JE. Anosognosia increases caregiver burden in mild cognitive impairment. Int J Geriatr Psychiatry. 2016 Jul;31(7):799-808.

7 Yesavage JA, Brink TL, Rose TL, Lum O, Huang V, Adey M, et al. Development and validation of a geriatric depression screening scale: a preliminary report. J Psychiatr Res. 1982-1983-1983;17(1):37-49.
8 Galvin JE, Roe CM, Coats MA, Morris JC. Patient's rating of cognitive ability: using the AD8, a brief informant interview, as a selfrating tool to detect dementia. Arch Neurol. 2007 May;64(5):725-30.

9 Scharre DW, Chang SI, Murden RA, Lamb J, Beversdorf DQ, Kataki M, et al. Self-administered Gerocognitive Examination (SAGE): a brief cognitive assessment Instrument for mild cognitive impairment (MCI) and early dementia. Alzheimer Dis Assoc Disord. 2010 Jan-Mar;24(1):64-71.

10 Gilewski MJ, Zelinski EM, Schaie KW. The Memory Functioning Questionnaire for assessment of memory complaints in adulthood and old age. Psychol Aging. 1990 Dec;5(4): 482-90.

11 Zelinski EM, Gilewski MJ, Anthony-Bergstone CR. Memory Functioning Questionnaire: concurrent validity with memory performance and self-reported memory failures. Psychol Aging. 1990 Sep;5(3):388-99.

12 Ura C, Miyamae F, Sakuma N, Niikawa H, Inagaki $\mathrm{H}$, Ijuin $\mathrm{M}$, et al. [Development of a selfadministered dementia checklist (SDC) (1): examination of factorial validity and internal reliability] [Article in Japanese]. Nippon Ronen Igakkai Zasshi. 2015;52(3):243-53.

13 American Psychiatric Association. Diagnostic and Statistical Manual of Mental Disorders (DSM-IV). 4th ed. Washington (DC): American Psychiatric Association; 1994.
14 Galvin JE, Roe CM, Powlishta KK, Coats MA, Muich SJ, Grant E, et al. The AD8: a brief informant interview to detect dementia. Neurology. 2005 Aug;65(4):559-64.

15 Galvin JE, Roe CM, Xiong C, Morris JC. Validity and reliability of the AD8 informant interview in dementia. Neurology. 2006 Dec; 67(11):1942-8.

16 Meguro K, Kasai M, Nakamura K; Kurihara Project members. [Reliability and validity of the Japanese version of the AD8] [Article in Japanese]. Nippon Ronen Igakkai Zasshi. 2015;52(1):61-70.

17 Hughes CP, Berg L, Danziger WL, Coben LA, Martin RL. A new clinical scale for the staging of dementia. Br J Psychiatry. 1982 Jun;140(6): 566-72.

18 Meguro K. The CDR Scoring Handbook for Early Detection of Dementia. Tokyo: IgakuShoin; 2008.

19 Hanley JA, McNeil BJ. A method of comparing the areas under receiver operating characteristic curves derived from the same cases. Radiology. 1983 Sep;148(3):839-43.

20 Bland JM, Altman DG. Cronbach's alpha. BMJ. $1997 \mathrm{Feb} ; 314(7080): 572$.

21 Kasai M, Meguro K. Patients with very mild dementia may confuse objective cognitive impairments with subjective physical health of quality of life: The Tome City Project in Japan. Front Psychol. 2018 Apr;9:533. 